\title{
Problematizar: acción fundamental para favorecer el aprendizaje activo $^{2}$
}

\author{
Problematize: Vital Action to Favor Active Learning
}

\section{Resumen}

La problematización es una condición fundamental del aprendizaje activo, en el que los alumnos son actores principales, pues aprender es un proceso llevado a cabo, en primera persona, por el sujeto, mediante la acción sobre los objetos de aprendizaje y el concurso de diversas dimensiones del aprendiz (volitivas, emocionales, relacionales, cognitivas, etc.). $\mathrm{Al}$ considerar la responsabilidad docente de diseñar y disponer en el aula de estrategias que favorezcan el aprendizaje, se habla de la acción de problematizar, la cual es considerada una estrategia didáctica que hace posible la problematización. En el presente trabajo se pretende responder a la pregunta: ¿cuáles son las características de una actividad de enseñanza/aprendizaje para que sea una acción de problematizar? Se considera que esta precisión teórica puede facilitar el trabajo de la investigación empírica, en la medida que establece los atributos esta estrategia didáctica. Asimismo, puede cualificar la actividad docente, pues ofrece a los profesores un marco de referencia para la elaboración de actividades de aprendizaje y la evaluación de su actividad docente.

Palabras clave: Problematización, acción de problematizar, aprendizaje activo.

\begin{abstract}
To problematize is a vital condition of active learning where students are main characters because learning is a process carry out in first person, by the subject through the action over learning objects and the participation of a diverse learner dimensions (volitive, emotional, relational, cognitive, etc.). Taking into account the responsibility of teachers of design and propose into the classroom the strategies that favor learning it is evident the action of problematize which is considered a didactic strategy that makes possible the problematization. This paper answers to the question what are the characteristics of a teaching-learning activity to be a problematize action? It is considered that this theoretical accuracy can make easier the task of empirical research since it determines the characteristics of this didactic strategy. Equally, this paper can qualify teaching tasks because it sets up a framework to carry out learning activities and teachers'self-evaluation.
\end{abstract}

Keywords: Problematization, action of problematize, active learning.

Recibido: 17 de marzo de 2014, evaluado: 15 de mayo de 2014, aprobado: 28 de mayo de 2014

1 Licenciado en Teología y Psicólogo. Posee formación en Ciencias de la Educación a nivel de pregrado y de maestría. Ha sido docente en la Facultad de Teología de Fortaleza (Brasil), en el Seminario Mayor San Carlos Borromeo de Cartagena y en el programa de Psicología de la Facultad de Ciencias Humanas y Sociales - UNIMINUTO, Sede Principal.

2 Artículo de reflexión teórica derivado de un proceso de investigación en el contexto de la Corporación Universitaria Minuto de Dios, que pretende indagar sobre las características de los procesos de enseñanza/aprendizaje en lógica de la acción de problematizar. 
$\mathrm{E}$ 1 presente artículo de reflexión tiene como contexto inmediato la Corporación Universitaria Minuto de Dios (Uniminuto), en su sede principal. Esta institución establece explícitamente que ha optado por un modelo educativo centrado en la formación integral, entendida como la educación que pretende el desarrollo armónico de las diversas dimensiones de la persona. En tal sentido, el saber (teoría) y el actuar (praxis) se articulan en el proyecto personal de vida de cada alumno, el cual se concibe como su manera idiosincrática y socialmente responsable de realizarse como persona y profesional (Uniminuto, 2004).

El modelo se desarrolla en tres campos de formación: desarrollo humano, práctica social y profesional, y competencias profesionales específicas. Para esto se requiere que los docentes de la institución, además de poseer los conocimientos y capacidades propias de su disciplina o profesión, desde el punto de vista pedagógico y didáctico, sepan "enseñar" a sus estudiantes a "aprender a aprender", en relación con los mencionados campos de formación (Uniminuto, 2004).

La característica general y fundamental del aprendizaje activo consiste en reunir las estrategias didácticas centradas en el proceso de aprendizaje de los alumnos y los diversos procesos llevados a cabo por éstos en dicha actividad. Sin embargo, no se trata de una manera de proceder automática, no intencionada. Al contrario, de acuerdo con Huber (2008), las diversas estrategias del aprendizaje activo confrontan tanto a estudiante como a profesores, ${ }^{3}$ con la dificultad común de asumir las exigencias de tal postura. Esto es, la necesidad de que profesores y alumnos se dispongan intencionalmente a la asunción de la centralidad efectiva de los alumnos en el proceso de la enseñanza y el aprendizaje.

En el presente trabajo se pretende establecer los aspectos constitutivos de la acción de proble- matizar. Esto es, responder a la pregunta: ¿cuáles son las características de una actividad de enseñanza/aprendizaje para que sea una acción de problematizar? Se considera que esta precisión teórica puede facilitar el trabajo de la investigación empírica, en la medida que establece los atributos de la estrategia didáctica en cuestión. Asimismo, puede cualificar la actividad docente, pues ofrece a los profesores un marco de referencia para la elaboración de actividades de aprendizaje y la evaluación de su actividad docente, pretendidamente problematizadoras.

En las páginas siguientes se exponen, en primer lugar, las características generales del aprendizaje activo, en cuanto propuesta teórica y metódica. En seguida, se considera la acción de problematizar, que incluye una definición de esta acción, un análisis del problema y la problematización en el campo didáctico y la síntesis de las propuestas que sobre este asunto tienen algunos pedagogos y la psicología cognitiva. Por último, se exponen las características de la acción de problematizar, que se derivan de los temas tratados en los puntos anteriores.

\section{El aprendizaje activo}

La expresión aprendizaje activo refleja una teoría sobre cómo se aprende al hacer, experimentar e interactuar con las personas y los diversos contenidos del ambiente, y cuando la actividad tiene como finalidad el significado y la comprensión (Schawartz y Pollishuke, 1998). Según esto, se considera que los alumnos son actores principales en el proceso de enseñanza y aprendizaje. Para lo cual es requerida la implicación motivacional, atencional y volitiva de los aprendices (Huber, 2008; Kristensen, Sander-Regier, Merhy y McColl, 2007; St-Jean, 2001).

Desde el punto de vista de las ciencias de la educación como la pedagogía, la psicología edu-

3 A lo largo del artículo se utilizarán indistintamente los términos docente, profesor y educador. 
cativa, la historia de la educación o la filosofía educativa, se ha dado un amplio reconocimiento a un cambio de paradigma en el proceso de enseñanza/aprendizaje. Ahora se le concede el lugar central al estudiante o alumno, concebido como actor principal, por tanto como aprendiz, en el desarrollo de este proceso. Sin embargo, la asunción de la centralidad del aprendiz, aunque parezca una novedad, parece obedecer a una "necesidad urgente", evidenciada hace decenios por eminentes pedagogos. Precisamente, Huber (2008) sostiene que en diversos pasajes de las obras de Cropley, Knowles, Treffinger, Barton, Weltner, Gaudig y Piaget se hace referencia a un tipo de aprendizaje, necesario por demás, que sustancialmente corresponde a lo que hoy en día se llama aprendizaje activo.

En el contexto anglosajón, Marquardt y Waddill (2004) afirman que el concepto de aprendizaje activo fue introducido por Reg Revans en la década del cuarenta. A partir de entonces, afirman estos autores, se han presentado diversas variaciones del concepto. Sin embargo, el aprendizaje activo comparte los elementos empleados en la práctica de las personas para resolver y actuar en problemas en tiempo real y aprender a través del cuestionamiento y la reflexión mientras hacen esto.

Es difícil establecer una definición exhaustiva sobre el aprendizaje activo. Revans siempre fue reticente a dar una única definición; parece que procuraba evitar que el aprendizaje activo se convirtiera en un simple procedimiento, de esta manera preservaba su aspecto filosófico (Barbosa, Batista, Baggio \& Kleinubing, 2012; Marquardt \& Waddill, 2004). Según Marquardt y Waddill (2004), aunque Revans nunca haya operacionalizado el aprendizaje activo en un modelo estándar, es posible señalar los principales esfuerzos por recoger los elementos esenciales y críticos, entre éstos considera el trabajo de Dilworth, 1998; Dotlich y Noel, 1998; Marquardt, 1999 y 2004; Mumford, 1991; Pedler, 1997; Weinstein, 1995.

De acuerdo con Marquardt (1999 y 2004), algunas de las características fundamentales del aprendizaje activo son los problemas en tiempo real, la relación sobre las acciones y resultados, el trabajo en equipo, el contexto de trabajo, los cambios de perspectivas y las experiencias compartidas. Se trata de un abordaje estrechamente relacionado con la resolución de problemas que contribuye al desarrollo de competencias a través de la adquisición de conocimientos e intercambio de experiencias.

Marquardt (2004) considera seis constructos principales del aprendizaje activo: el problema, el grupo, los cuestionamientos, la acción, el apoyo y el aprendizaje. La interrelación de estos componentes constituye el aprendizaje activo. Ser parte de un problema, que es abordado por el grupo, con el fin de encontrar soluciones, para tal fin se generan diversas preguntas y se pasa a la acción. En dicho proceso, con la guía del educador, los alumnos adquieren un aprendizaje significativo, esto es, sustancial y duradero. Además desarrollan o adquieren destrezas y habilidades respecto a la adquisición de las informaciones y a los métodos empleados.

En definitiva, según Marquardt y Waddill (2004), el aprendizaje activo se construye a partir de un problema, cuya resolución es altamente importante para un individuo o grupo. El problema debe ser significativo, esto es, encontrarse dentro de las responsabilidades del equipo o individuo que lo resuelve y ofrecer la ocasión para el aprendizaje.

Según Shuell (1986), citado por Huber (2008), el aprendizaje activo (para enfatizar que nadie aprende en el lugar de otra persona, sino que cada quien aprende por sí mismo) sería uno de los rasgos que caracteriza los procesos de educación/ formación orientados a los estudiantes y su participación activa en ellos. Los otros rasgos serían el aprendizaje autorregulado (para referirse a la capacidad de percibir las propias actividades, los resultados de las mismas y la retroalimentación para mejorar), el aprendizaje constructivo (en el que se concibe el conocimiento como una construcción personal), el aprendizaje situado (que reconoce la relevancia del contexto, tanto para la 
aplicación como para la adquisición de los conocimientos) y el aprendizaje social (pues aprender no es un proceso exclusivamente individual).

En este enfoque el profesor deja de ser el detentor y dispensador del conocimiento para transformarse en guía, que orienta los aprendices; estratega que dispone actividades que permitan el aprendizaje activo de los aprendices; asesor que aclara dudas, ante informaciones o problemas que superan el grado de competencia de los aprendices (Bozu \& Canto, 2009).

El proceso de enseñanza y aprendizaje, según las características del aprendizaje activo, implica didácticas en las que los alumnos son actores principales del aprendizaje. Tal presupuesto ha dado lugar a diversos métodos de enseñanza/ aprendizaje. Huber (2008) reporta el método de proyectos, en el que se enfatiza en la unificación del aprendizaje teórico y práctico, el aprendizaje colaborativo y la inclusión de elementos de la vida cotidiana; el método de aprendizaje basado en problemas, cuya finalidad se orienta al aprendizaje sobre cómo descubrir el núcleo problemático de una situación, y al cómo aprender; y los métodos WELL (Wecheslseitiges Lehrenund Lernen) que promueven la colaboración entre los alumnos y la adquisición de estrategias de aprendizaje.

Según Huber (2008), la reflexión es el método general del aprendizaje activo. Implica dirigirse al objeto de aprendizaje y a los procesos que lo hacen posible, incluso la misma la reflexión. Sin embargo, este proceso implica la problematización como método que permite involucrar y motivar al alumno (Minardi, Siqueira, Girardi, Morais, Almeida, Pinto, Moreira y Amaral, 2008). No basta con hacer referencia a un problema, se requiere la activación de una acción relativa al problema: la acción de problematizar.

A partir de lo expuesto en los párrafos anteriores se concluye que una determinada estrategia didáctica, que se pretenda representativa del aprendizaje activo, debe dar lugar a procesos de reflexión y problematización, en los cuales los alumnos realicen un aprendizaje significativo y desarrollen las competencias que les permitan aprender a aprender. A continuación se definen la acción de problematizar y sus características.

\section{La acción de problematizar}

Términos como problematización, problemática, problematizar, derivados de problema, se han difundido en los escritos académicos. Sin embargo, según señala Benoit (2005) no se encuentran desarrollados en los diccionarios especializados de filosofía y educación. Se trata de neologismos, afirma este autor, en el contexto francófono, que han tenido una gran acogida, aunque es necesario todavía aclarar su significado. Son por tanto conceptos en proceso de definición, sobre las cuales es necesario precisar el sentido con el cual son utilizados.

Se parte de la expresión acción de problematizar en lugar de problematización, debido al hecho de que problematizar corresponde a un verbo, por tanto, a una acción que debe ser actuada por un sujeto. Se considera a los profesores, en el contexto educativo, quienes realizan tal actividad, en la medida que organizan o presentan una determinada actividad bajo la forma de un problema. Sin embargo, las implicaciones de esta actividad provienen de la específica concepción que se tenga de lo que es problema y problematización.

La gran mayoría de los investigadores en didáctica de la ciencia están de acuerdo en que la adquisición de saberes científicos no puede consistir simplemente en acumular de manera no problemática informaciones sobre el mundo. De ahí la importancia de considerar el problema y la problematización en el campo didáctico. El problema es frecuentemente justificado por referencia a lo que hacen los investigadores o por ser un medio para fragilizar o transformar preconcepciones de los alumnos o por ser la oportunidad para adquirir un método (Orange, 2005).

Al tratar el lugar de los problemas y la problematización en la didáctica, Orange (2005) consi- 
dera tres dimensiones de estudio: las referencias epistemológicas y psicológicas; el tipo de problemas asumidos por los alumnos; el tipo de descripciones de los procesos de trabajo del problema y la problematización.

En general, un buen número de epistemólogos contemporáneos atribuye al problema una función mayor en el funcionamiento de las ciencias. Según Orange (2005), se trata de romper con una descripción empirista o positivista de la actividad científica; sostiene, además, que epistemólogos racionalistas como Gaston Bachelard y Karl Popper consideran que los problemas son el punto de partida del conocimiento y que la relación problemas-conocimientos también es considerada por pragmatistas, como John Dewey.

Esta doble referencia, pragmatista y racionalista, es estudiada por Fabre (2005). Según este autor, Dewey y Bachelard sitúan el problema en una ambiente con una gran riqueza de significado, ya sea la complejidad de la vida (Dewey) o la investigación científica (Bachelard). Un problema es puesto cuando es percibido y asumido por un sujeto. Pero todavía se debe construir, esto es, reunir los datos y las condiciones, después resolverlo poniendo a prueba las hipótesis de solución. Las dos posturas epistemológicas insisten, cada una a su manera, sobre la construcción de problemas y no solo sobre su resolución.

Explica Fabre (2005) que se trata de una crítica al empirismo, en cuanto conllevan un rechazo a todo conocimiento inmediato. Para Dewey, continúa Fabre, todo saber resulta de una pesquisa. Pero en una investigación la base del cuestionamiento se presenta como proposición independiente. Se olvida entonces que ella no constituye sino la conclusión de una pesquisa precedente. Hay ahí un proceso de fetichización del conocimiento al cual, según Fabre, Bachelard se opone también. Se requiere, por tanto, la discusión (problematización) para que surja la originalidad del conocimiento. Se podría decir que para estos dos filósofos el conocimiento no consiste en el ver, sino en el trabajar y discutir.
Orange (2005), tras examinar los parcos teóricos empleados por las investigaciones que se ocupan de los problemas y la problematización, concluye que no hay unidad teórica sobre este asunto. Sin embargo, el constructivismo, todavía muy genérico, está presente como telón de fondo común, pues encuentra referencias que implican diversas combinaciones entre, por ejemplo, Bachelard, Popper, Piaget, Vygostki, Dewey, entre otros.

La diversidad de marcos teóricos tal vez se puede explicar por tratarse de diferentes problemas de estudio. Éstos no tienen el mismo estatus epistemológico y no tienen la misma forma didáctica. Según Orange (2005), en principio se pueden distinguir entre los problemas de naturaleza exclusivamente científica y los que corresponden a situaciones socialmente vivas.

Si, por ejemplo, los problemas de la actividad escolar se refieren a la cuestión de la peligrosidad del uso de los teléfonos celulares o si los parques nacionales son una solución para el mantenimiento de la biodiversidad, se trata de situaciones socialmente vivas. En tal caso, dice Orange (2005), se trata de situaciones que no tienen una única solución, con algunos componentes inciertos. Éstas no implican sólo saberes técnico-científicos, sino consideraciones éticas, económicas, políticas. Además de ser situaciones todavía discutidas por la comunidad científica.

Por otra parte, si los problemas se refieren, por ejemplo, a las especies propias de un determinado lugar, entonces se está trabajando con un problema esencialmente científico. En éstos, aclara Orange (2005), se hace una adaptación didáctica de cuestiones generalmente resueltas por la investigación de la comunidad científica y que generalmente privilegian problemas relevantes del análisis descriptivo.

Sin embargo, esta clasificación de los problemas es seguramente discutible a partir de las posturas epistemológicas en las que la descripción de la actividad científica no se deslinda de los aspectos sociales, internos o externos. Por ejem- 
plo, aclara Orange (2005), el paradigma de Kuhn. Rumelhard (2005), al considerar este asunto específicamente la cuestión didáctica de la construcción de problemas, afirma que los alumnos piensan en un espacio-tiempo cultural determinado, el cual frecuentemente afecta, aunque no siempre se diga, la formulación de los problemas.

Los problemas también pueden ser considerados en razón de la forma en que son usados en el contexto escolar. Orange (2005) presenta la investigación de Boilevin (2005), en la que hay una clara distinción entre situaciones-problema y problemas abiertos. La situación problema se centra en la superación de un obstáculo y las indicaciones son precisas, de tal manera que sea necesario aprender para poder lograr el objetivo. En los problemas abiertos, por otra parte, se busca sobretodo el aprendizaje del método científico, su referencia es la actividad de los investigadores, por eso, los datos del problema no están claramente preestablecidos por el profesor.

Sin embargo, advierte Orange (2005), esta distinción no es muy precisa. Algunas veces se habla de situación-problema, pero los objetivos y la organización del problema corresponde más a la descripción que se ha hecho de problema abierto. En todo caso, según Orange (2005), una buena parte de los usos didácticos del problema aparecen explícitamente relacionados con el debate en clase.

La tercera distinción expuesta por Orange (2005) corresponde a la problematización a que da lugar el problema, esto es, la manera como es asumido en la actividad de los alumnos. Por una parte, la problematización corresponde al desarrollo de un cuestionamiento, se trata de seguir un modelo de tratamiento del problema. Por otra parte, otras maneras de abordar el problema enfatizan la definición de los alumnos. Este punto de vista se encuentra más cercano a los postulados de la psicología cognitiva, bien sea porque el alumno expresa las cuestiones en términos de conceptos científicos o porque hacen una elaboración colectiva del conjunto de las representaciones en controversia.
Se puede apreciar, según lo expuesto sobre el problema y la problematización, la diversidad conceptual y metodológica sobre el asunto. De ahí que la acción de problematizar, entendida como la actividad del docente mediante la cual presenta el problema a los alumnos para que acontezca un abordaje del mismo, tenga realizaciones diversas, de acuerdo al marco de referencia desde el cual se esté actuando. Sin embargo, también sobresale en los análisis anteriores la posibilidad práctica de integrar esta diversidad, supuesto que en todas ellas el conocimiento no corresponde principalmente a la acumulación de informaciones dadas, sino a la construcción de conocimiento mediante la problematización, discusión y reflexión.

Zanotto y Rose (2003), a partir de los aportes de Dewey, Saviani, Freire y de la psicología cognitiva sobre la problematización, concluyen que, más allá de las diferencias teóricas que puedan existir entre las fuentes consideradas, hay coincidencia en cuanto a la definición de la acción de problematizar. Ésta consiste en saber relacionar de forma coherente y secuenciada: (1) la identificación de un problema, que es relevante, específico y objetivo; (2) la búsqueda de los factores que explican el problema de forma suficiente y pertinente; y (3) la generación de soluciones. Los aportes teóricos tenidos en cuenta para para la elaboración de la anterior definición se exponen a continuación.

\section{La problematización en Dewey}

Según Westbrook (1993), John Dewey (1859-1952) es considerado el filósofo estadunidense más importante en los primeros 50 años del siglo XX; su filosofía abogaba por unidad entre la teoría y la práctica. En el campo educativo es considerado como precursor de la enseñanza "centrada en el niño". En la madurez de su pensamiento pasó gradualmente del idealismo al pragmatismo y naturalismo. Desde esta perspectiva, el pensamiento adquiere el carácter de conocimiento cuando se le comprueba en la acción, al resolver 
problemas. El aprendizaje ocurre, precisamente, para adultos y niños al enfrentar situaciones problemáticas.

Fabre (2006), para responder a la cuestión acerca de cómo la teoría de Dewey puede ayudar a comprender lo que quiere decir problematizar, en la escuela y en la formación, hace un análisis detallado de los planteamientos de este autor. El punto de partida es la comprensión del ser humano como un organismo en situación. La interacción entre el organismo y el ambiente se comprueba ontológicamente primero. Para pensar en las múltiples interacciones del individuo es necesario ocuparse de la situación que constituye la individualidad verificable. La situación constituye el conjunto de las interacciones entre el sujeto y el ambiente, o incluso, esté experimentado por el sujeto.

Para Dewey, expone Fabre (2006), la experiencia se entiende como ajuste adaptativo, en el que se puede reconocer una dialéctica de pasividad y actividad. En tal sentido, la experiencia se define más como aprendizaje que como conciencia. Tal posición se puede aplicar a cualquier ser vivo. Sin embargo, hay una variación en el grado de actividad sobre las pruebas y en la anticipación de la acción sobre el medio.

La idea de situación en Dewey, según la exposición de Fabre (2006), va contra el idealismo, pues tiene una base biológica como forma superior de adaptación, pero sin caer en el reduccionismo, pues se considera la especificidad de los intereses intelectuales. Además, esta idea, en el sentido pragmático, corresponde a una posición original entre el empirismo y el racionalismo clásico. La mente no es un tablero en blanco: las ideas provienen principalmente de las experiencias anteriores superadas.

La vida biológica y cultural de los sujetos se constituye de equilibrios y desequilibrios. Hay un problema cuando se percibe un desequilibrio que no se puede remediar de manera inmediata y es necesario encontrar una solución. Se pueden considerar dos categorías: los que nos sobrepasan porque son completamente reales y no nos dejan salida, pues su presión no nos deja agarrarnos a nada conocido. Pero hay problemas que, aunque son inminentes, dejan espacio porque podemos hacer inferencia a partir de lo ya conocido. En todo caso, el sujeto debe hacerse cargo del desequilibrio que experimenta e intentar reducirlo. Al ser problemática una situación, ella hace necesaria la investigación y pensar en el problema (Fabre, 2006).

El tratamiento de los problemas, precisa Fabre (2006), recibe en Dewey el término genérico de investigación, con el cual se refiere a la transformación controlada y dirigida de una situación indeterminada a una situación determinada en sus distinciones y relaciones constitutivas. Construir un problema implica articular referencias (datos) e inferencias (sugerencias, hipótesis). Las referencias designan: (a) lo que está realmente presente en la situación; (b) lo que constituye una molestia, que me veo obligado a considerar; y (c) lo que se considera conocido. Las inferencias se refieren a: (a) lo que está por llegar, que no está realmente presente en la situación; (b) lo que tiene el carácter de posible; y (c) lo que es desconocido, pero se puede inferir a partir de lo conocido. En la medida que se avanza en la investigación las inferencias se transforman en referencias.

¿Cómo progresa la investigación? Es necesario concebir las referencias y las inferencias de manera constructivista: todo es construido por el sujeto que trata el problema. Debe encontrar las referencias e imaginar las inferencias. Al respecto, Dewey interpreta el sentido lógico de la negación y la afirmación considerándolas funciones que buscan seleccionar los elementos pertinentes del problema. Se da una sucesión de referencias e inferencias, las que surgen por el crecimiento de aquellas (Fabre, 2006).

La serie de referencias constituye el material de la investigación. La situación se determina en la medida que se cuenta con el inventario de datos completo. A la operación de preparar el material Dewey le da el nombre de inducción, que no equivale al paso de lo general a lo específico, 
sino a la consecución de un esquema inteligible a partir del análisis de todos sus componentes. La función de las inferencias consiste en aportar nuevos materiales y relacionar los ya existentes. No se trata simplemente de hacer una lista, sino de ofrecer un proyecto (Fabre, 2006).

En cierto modo, las referencias son siempre del orden de los hechos y las sugerencias del orden de las ideas. El conocimiento proviene tanto de las sensaciones como de las ideas y las ideas no son copias de las sensaciones, sino anticipaciones, programas de experiencia, son del orden de los proyectos. Aunque tal formulación parezca kantiana, diverge en tanto que las categorías no son elementos de un intelecto inmutable, sino que permanecen tributarias de una historia, por lo tanto cambiantes; y que no corresponde a síntesis, la única articulación entre conceptos e intuiciones es el dinamismo de la investigación (Fabre, 2006).

Según Fabre (2006), esta aproximación hace progresar el análisis al incluir en la investigación una teoría general del juicio. Esto obliga a Dewey a hacer una audaz reformulación de la proposición lógica. Además, aquí las sugerencias no obedecen a la esfera del sentido común y a los conocimientos cotidianos, sino a los significados culturales especializados. Se retoma la tradición racionalista en la que pensar es juzgar, en el sentido de relacionar un sujeto y un predicado. Pero, para Dewey la noción es más amplia, los componentes del juicio no son dados, sino construidos por la investigación. Tanto la definición del sujeto, como lo que se predica de él y la relación que se establece son el resultado de un proceso de investigación que ha permitido suprimir un desequilibrio existencial.

En síntesis, se subrayan tres rasgos en este abordaje. En principio, Dewey se opone a la concepción subjetivista del problema. No se trata solamente de un asunto psíquico, el problema está en el mundo. Tampoco es un asunto escolástico, no se reduce simplemente a una dificultad intelectual, se trata siempre de un desequilibrio existencial que se debe solucionar. Además, en el abordaje del problema se distinguen diversas fases o dimensiones del problema: la posición, la construcción y la resolución.

Se puede deducir de la exposición realizada el carácter activo del sujeto que aprende, sobre todo al ser concebido como investigador. Respecto al proceso de la enseñanza y el aprendizaje se deben proponer auténticas situaciones de experiencia, que requieran alcanzar un saber que lo sea efectivamente, ante la naturaleza del problema. Para esto es necesario poner en marcha procesos reflexivos relacionados con la construcción de referencias y de inferencias y con el proceso de juicio.

\section{La problematización en Dermeval Saviani}

Este filósofo y educador brasileño, considerado como el idealizador de la pedagogía históricocrítica, expone el papel de la problematización en la educación. En primer lugar señala que generalmente se entiende como problema, pero que no constituye auténtico problema. Tal es el caso de la cuestión o pregunta, del misterio, el enigma, el obstáculo, la dificultad o la duda (Saviani, 1996).

Propone entonces la necesidad de recuperar la problematicidad del problema. Saviani (1996) afirma que el ser humano se habituó a vivir en el problema, es decir, a estar en una situación en la que no puede permanecer más y por eso exige una solución. Se trata entonces de una situación vital signada por la necesidad y en la que no es posible continuar sin encontrar una solución. Esta es la esencia del asunto.

Se aprecia que el problema tiene un sentido profundamente vital y altamente dramático. Se refiere a una situación de impase. Esto, sin embargo, no equivale a mera subjetividad. Aunque es vivido de manera subjetiva, la situación que se constituye en problema para alguien lo es porque existen condiciones objetivas reconocibles y determinables. 
Encontrar la solución requiere de la reflexión. En efecto, se establece una relación dialéctica en reflexión y problema, en la que se debe evitar privilegiar cualquiera de estas dimensiones. Si se lo hace con aquella se cae en el subjetivismo, en la presunción de que el ser humano tiene poder casi absoluto sobre los problemas y que puede manipularlos a su antojo. Si se lo hace sobre éste se cae en la reificación, con lo cual se lo desvincula de su estricta relación con la existencia humana, sin la que su esencia no puede ser aprehendida, como se explicó antes.

De acuerdo con esta exposición, el alumno es reconocido como sujeto que conoce, el cual actúa como tal en la medida que se enfrenta a problemas que no son meros obstáculos o preguntas retóricas, sino situaciones que le exigen el hallazgo de una solución. Para tal fin, es menester que intervenga la reflexión como actividad que permita dilucidar las condiciones subjetivas y objetivas de la situación problema y la puesta en marcha de las estrategias de solución.

\section{La problematización en Paulo Freire}

Paulo Freire (1921-1997) es un pedagogo brasileño, conocido por sus esfuerzos por la alfabetización de los adultos en medios pobres y la alfabetización como militancia contra la opresión. Para él, la educación es un proceso de concienciación y liberación.

La reflexión y la problematización son nucleares en el quehacer del profesor, en cuanto sujeto que enseña, pues enseñar implica una acción transitiva y relativa en la que los sujetos no se pueden convertir uno en objeto del otro; por tanto, no es transferir conocimiento, sino crear las posibilidades de su producción o de su construcción. Enseñar como tarea incrustada en el aprender implica encender en el aprendiz la actitud crítica en el proceso de aprendizaje, lo cual posibilita la curiosidad epistemológica (Freire, 2006).
La capacidad humana de aprender, que hace posible la de enseñar, implica la habilidad de aprehender la sustantividad que aquello que se aprende. Por tal motivo, la memorización mecánica o la recepción de un perfil del objeto o del contenido no es un verdadero aprendizaje. En tal caso, se reduce el aprendiz a la condición de paciente en un proceso de transferencia y se le niega la condición de sujeto crítico, epistemológicamente curioso, que construye el conocimiento o participa en su construcción (Freire, 2006).

Para Freire (2006) es fundamental la conciencia del carácter inacabado del ser humano. Tal condición remite a su carácter histórico y existencial, a la libertad como decisión acerca de su manera de existir y la ineludible dimensión ética del acontecer humano. Inacabado quiere decir no absolutamente determinado y, por lo tanto, facultativo, aunque históricamente condicionado. De tal supuesto se deriva la tarea de propiciar las condiciones para que los educandos en sus relaciones entre sí y de todos con el profesor o profesora puedan ensayar la experiencia de asumirse en su carácter existencial, histórico y ético.

Se aprecia en la postura de Freire que enseñar y aprender no son actividades neutras, exclusivamente intelectuales u operativas, sino que implican la dimensión formativa de la educación, en cuanto pide la reflexión y transformación constante de la manera de existir en el mundo y con los otros.

Entonces, por consistir en un acto de conocimiento y en actividad formadora, lo fundamental es que profesores y alumnos sepan que la postura que los caracteriza en el proceso de enseñanza y aprendizaje es dialógica, abierta, curiosa y no pasiva. Para tal fin, cabe al profesor estimular la reflexión crítica y la problematización en los alumnos, lo cual no consiste simplemente en preguntas retóricas con respuestas, que no lo son, a preguntas que nunca fueron hechas.

La problematización se da en el terreno de la comunicación, alrededor de situaciones reales o en torno a los contenidos intelectuales, que tam- 
bién se refieren a lo concreto, y pide la comprensión de los signos significantes presentes en los significados por parte de los sujetos problematizados. Por tanto, la problematización acontece en el diálogo y la comunicación que hace posible la comprensión de los términos expresa el análisis crítico del problema que los compromete (Freire, 1983).

La problematización está unida al acto cognoscente así como a las situaciones concretas. Parte de éstas, incluso cuando el problema parece netamente intelectual, pues los contenidos intelectuales permanecen referidos al acontecer histórico e implica un retorno crítico a la acción. En el fondo, la problematización equivale a reflexión sobre un contenido, que el resultado de un acto, o sobre el acto, con el fin de actuar mejor en relación con los otros y con la realidad. Por tanto, Freire defiende una concepción educativa referida a la problematización del "hombre-mundo", del hombre como existente, ineludiblemente responsable de su manera de estar en el mundo (Freire, 1983).

Aunque sería posible estudiar la problematización desde diversas perspectivas, para Freire (1983) lo que importa fundamentalmente en la educación como situación de conocimiento es la problematización del mundo, de la cultura y de la historia, en cuanto resultado de la relación entre el hombre y el mundo, que condiciona a los propios hombres.

Para Freire (1983) la actividad educativa corresponde a un proceso de admiración del mundo (mundo construido en la relaciones humanas) y readmiración de las miradas pasadas. Los seres humanos, alumnos y profesores son sujetos de conocimiento y no recibidores de un conocimiento que otros les entregan o prescriben. Su condición de sujetos activo implica ir ganando la "razón" de la realidad, la cual, precisamente por este conocimiento, se revela como un mundo de desafíos y posibilidades. En este sentido, la educación corresponde a un quehacer que permanece, que está siendo, el cual implica una dinámica transformadora.
En síntesis, la problematización es una actividad reflexiva que acontece en la comunicación. Como proceso, parte de una situación problema, la cual es admirada y readmirada; que requiere soluciones provistas de valor cognitivo, práctico y ético. En efecto, el quehacer problematizador es totalizante, la situación problema se inscribe en el mundo humano, por esto requiere ser aprendida críticamente como parte de una realidad procesual mayor.

\section{La problematización en la psicología cognitiva}

El marco teórico general de la problematización es el enfoque constructivista del aprendizaje. Sin embargo, en líneas generales este enfoque del aprendizaje, como se verá a continuación, se basa fundamentalmente en los aportes de la psicología cognitiva del aprendizaje. Asunto que se aborda en este momento con el fin de configurar las características de la problematización, de acuerdo a los aportes de estos enfoques del aprendizaje.

Chadwick (2001) advierte la inexistencia de una comprensión unívoca del constructivismo en la educación, pues este aún no refiere una teoría comprensiva de los procesos de enseñanza y aprendizaje. Sin embargo, es posible congregar diversas teorías y explicaciones provenientes de la psicología cognitiva, a partir de las cuales este autor busca los lineamientos que permiten entender este enfoque. Precisamente, reconoce a Baldwin, Dewey, Piaget, Vygotski, Bruner y otros destacados teóricos e investigadores, entre los precursores del constructivismo. Análoga apreciación se puede encontrar en Ormrond, (2008).

En el planteamiento básico de este enfoque el aprendizaje corresponde a una construcción del sujeto, en la que además se construye a sí mismo, a partir de la interacción de sus disposiciones idiosincrásicas y el ambiente en el que se encuentra. De ahí que conocer no corresponde a adquirir una copia de la realidad, sino a una construcción 
de la misma. Al hacer esto, acontecen en el sujeto diversos procesos cognitivos referentes a la representación y procesamiento de la información en el ambiente, a la adquirida anteriormente y a la actividad desarrollada al respecto (Chadwick, 2001).

Este postulado del constructivismo involucra una específica consideración del sujeto cognoscente, del aprendiz, el cual realiza una actividad transformadora con relación a la información ante la cual se encuentra, la revisa, la clasifica, la asimila, etc. Para esto es indispensable que efectúe intencionalmente, de tal manera que no hay aprendizaje eficaz que no sea aprendizaje activo. Además, su actividad no se refiere exclusivamente a los contenidos (informaciones o representaciones del mundo circunstante), sino igualmente a la actividad realizada en el proceso de aprendizaje.

Esta consideración del aprendiz, como sujeto activo, protagonista del proceso de aprendizaje, podría erróneamente llevar a suponer que aprender es simplemente una actividad solipsista, reducida a simple descubrimiento autoguiado. Al contrario, el profesor continúa cumpliendo un papel fundamental, es guía que ayuda al aprendizaje, pero este es realizado en primera persona por el alumno. Para tal fin, cabe al profesor disponer las condiciones que favorecen la activación de los procesos cognitivos, de interacción con el ambiente, con otros interlocutores, etc., que hagan posible la realización de dicha actividad.

Se tiene, entonces, que el aprendizaje como construcción implica la generación de estructuras de conocimiento organizadas en cada sujeto a partir de las interacciones previas (experiencias), las cuales sirven de ancla a nuevos conocimientos, que a su vez reconfiguran la estructura cognitiva preexistente (Ausubel, Novak \& Hanesian, 2006). Además, puesto que este proceso requiere la interacción con los otros, mediado por los dispositivos de comunicación de cada cultura, el aprendizaje es un producto de la interacción social y cultural (Vygotski, 1989).

Un aspecto importante en el proceso de aprendizaje, según es expuesto por las teorías de cogni- tivas (y constructivistas), es el estado de desequilibrio que mueve el alumno al aprendizaje (aporte de Piaget), pues se encuentra en una situación que ante la cual sus estructuras cognitivas previas resultan insuficientes para adaptarse a las nuevas circunstancias. Lo cual se articula con la teoría de la zona de desarrollo próximo (Vygotski), a partir de la cual el desafío que entraña este desequilibrio no puede superar las posibilidades, solución de que dispone el alumno, contando con la ayuda de los soportes socio-culturales a su alcance (Chadwick, 2001).

El énfasis puesto por el cognitivismo-constructivismo en la participación activa del alumno en el proceso de aprendizaje tiene como objetivo supremo el aprender a aprender. En ese caso, el aprendizaje no se refiere sólo a la adquisición de nuevas representaciones en la estructura cognitiva del sujeto, sino, además, a la adquisición de estrategias de aprendizaje, para lo cual la interacción se activa a procesos metacognitivos. Estos es, procesos de conocimiento referidos a los mismos procesos cognitivos que hacen posible el aprendizaje (Ormrond, 2008).

Puesto que se ha establecido que la problematización es una estrategia básica del aprendizaje activo, tal procedimiento didáctico se encuentra a la base de los procesos cognitivos y metacognitivos a los que se ha hecho referencia en los párrafos anteriores. Por tanto, estos procesos se deben activar en el alumno cuando acontece la problematización. Se trata de que el problema, como situación de impase (desequlibrio), al ser propuesto en el proceso de enseñanza-aprendizaje, lleve a buscar las informaciones y acciones necesarias para darle una solución.

\section{Características de la acción de problematizar}

A partir del análisis de los diversos abordajes sobre la problematización expuestos en los ítems anteriores y según la exposición de Zanotto y 
Rose (2003), se presentan los elementos constitutivos de la acción de problematizar.

1. La acción de problematizar implica, como se expuso antes, relacionar tres momentos: la identificación del problema, la búsqueda de los factores explicativos y la proposición de soluciones. Esta es una característica presente en los tres autores considerados, que para Zanotto y Rose (2003) constituye la estructura básica de la acción de problematizar.

2. El énfasis en el carácter activo del sujeto. Se trata de una característica sobresaliente en la exposición de Dewey. El sujeto, considerado como organismo vivo, biológica y socio-culturalmente situado, enfrenta el desequilibrio (problema), que suscita diversos procesos, asumidos en primera persona para la resolución de la situación. Con todo, se trata de una característica presente en los demás pedagogos estudiados, que además corresponde a la función básica de la problematización dentro del aprendizaje activo. Es el aprendiz quien debe pasar por los tres momentos mencionados antes, es él quien está ante una situación que se le vuelve un problema, quien debe examinarla y encontrar los factores explicativos y quien propone soluciones.

3. El énfasis en la problematicidad del problema. Es una característica especialmente señalada por Saviani. El problema no es equiparable a la duda, al cuestionamiento, al misterio, al enigma, etc. El problema, en términos de Dewey, como se vio en la exposición, es una auténtica situación vital que requiere solución. Es una exigencia de la condición histórica, como señala Freire, basada en la capacidad de respuesta del ser humano. Aunque el término problematicidad parezca excesivamente abstracto y perteneciente a la filosofía, antes que a la pedagogía o la didáctica, implica un aspecto del problema que se puede operacionalizar, en la medida que los alumnos, ante una situación dada, mani- fiesten que ésta efectivamente los confronta con la necesidad movilizarse en busca de una solución. En tanto que la situación propuesta no desequilibre al aprendiz y lo mueva a la superación de la misma, ésta no tendrá la condición de problema.

4. El énfasis en la praxis, como dimensión ética de la acción. El problema y la solución tienen una dimensión social y ética. Aunque Saviani y Dewey no excluyen la consideración del abordaje del problema y de las acciones encaminada a su solución, desde una perspectiva específicamente humana, es Freire quien enfatiza en este aspecto. Se trata de un tema central de su exposición. No es posible reconocer una situación como problema y moverse a la solución, sin que tales acontecimientos impliquen una transformación de la realidad y sin que esto conlleve una específica valoración ética. Freire enfatiza la dimensión existencial de la problematización, esto es, que en el acto de abordar un problema y buscar su solución, el sujeto cognoscente actúa como ser históricamente situado y, por tanto, el problema lo afecta no sólo a nivel cognitivo, sino incluso en su dimensión existencial. Esto es, en su libertad, en su manera histórica de configurarse como ser humano en el mundo.

5. La metacognición. En la acción de problematizar ocurre en el sujeto, los alumnos, un cambio respecto a la adquisición o transformación de estrategias de aprendizaje. Dewey y Freire señalan tal situación. Cuando un sujeto se enfrenta a una situación problema, que lo lleva a actuar procesos de aprendizaje, tal actividad es acompañada de la reflexión sobre los conocimientos adquiridos y los procesos actuados para alcanzarlo. El conocimiento sobre los propios procesos de aprendizaje es un aspecto característico de la concepción del aprendizaje a partir de la psicología cognitiva.

6. El cambio cognitivo. En la acción de problematizar también ocurre un cambio cogniti- 
vo. Se adquieren nuevos conocimientos, se perfeccionan o desechan los existentes. En las exposiciones de Dewey y Freire es central tal acontecimiento, el sujeto activo, es un sujeto cognoscente. Esto es así ya sea que se enfatice el carácter pragmático del proceso, en el caso de Dewey, histórico-crítico y existencialista, en el caso de Saviani y Freire, o cognitivo y constructivista.

\section{A modo de conclusión}

La acción de problematizar es la estrategia didáctica básica que debe llevar a cabo cualquier docente que pretenda generar un proceso de aprendizaje activo en los estudiantes a su cargo. Como se ha establecido en lo largo del presente artículo es fundamental que los profesores incluyan en la actividad docente situaciones que sean efectivamente problemas. Al respecto es imprescindible tener presentes las reflexiones de Saviani en lo referente a la naturaleza del problema, pues pudiera ocurrir que en su tarea los profesores se den por satisfechos al proponer actividades que no dan lugar a una auténtica problematización.

Los docentes nos deberíamos evaluar respecto a la calidad de las situaciones que proponemos como punto de partida del aprendizaje. Pudiera ocurrir que cuando los profesores hacen preguntas, estas tengan apenas una función retórica, sin que entrañen una auténtica situación ante la cual los estudiantes se deban mover a la búsqueda de una solución. Situación semejante puede acontecer cuando los educadores confrontan a los alumnos con misterios o enigmas, que pueden ser muy interesantes desde otros puntos de vista, pero no generan procesos de aprendizaje, antes enfatizan más la ignorancia humana o los límites del conocimiento.

Por otra parte, tanto los administradores de las instituciones educativas, como los docentes, deberían tener presente, según lo expuesto en este artículo, la relación indisoluble que existe entre actividad cognoscente y responsabilidad histórica. En otras palabras, la actividad educativa no consiste en la mera adquisición de conocimientos, pues éstos implican siempre una postura, una actitud, ante las circunstancias históricas en las que se encuentran los sujetos (profesores y alumnos).

A partir de lo dicho en el párrafo anterior, vale la pena dejar planteada la pregunta: ¿en qué medida la asignación de recursos, la evaluación de los docentes, la planificación en las instituciones educativas, entre otros aspectos institucionales, terminan concibiendo el proceso educativo como mera transmisión de información y desconociendo la necesidad del encuentro, la interacción humana, la reflexión, entre otros factores que eventualmente constituyen la tomada de decisiones de los educadores y estudiantes? Problematizar no se reduce a una técnica, aunque se haya expuesto un procedimiento, esta estrategia didáctica implica creatividad, cuestionamiento, transformación de las personas e instituciones.

Este estudio se propuso establecer las características de la acción de problematizar, pues, en la medida que los componentes o condiciones de esta estrategia didáctica se hacen explícitos y son reconocidos, los educadores $\mathrm{y}$ estudiantes tendrán mayores posibilidades de criticar y transformar sus prácticas de enseñanza/aprendizaje. En efecto, se espera que este artículo suscite otras reflexiones o investigaciones empíricas. Por ejemplo, determinar si actividades basadas en los principios teóricos expuestos efectivamente logran el propósito de un aprendizaje significativo, en el que los aprendices se reconocen actores del proceso de aprendizaje. También se podrían hacer estudios exploratorios en los que se examinan experiencias de enseñanza/aprendizaje consideradas exitosas, en cuanto que propician el aprendizaje activo, para verificar si las características encontradas corresponden a lo dicho en la teoría. 


\section{Referencias}

Barbosa, A., Batista, T., Baggio, A. \& Kleinubing, C. (2012). Dimensoes de um sistema de aprendizagem em acao para o ensino de administracao. Administracao: Ensino e pesquisa, 13(1). 9-41.

Benoit, J.P. (2005). L'émergence des "mots de la problématisation" dans les sources universitaires et les dictionnaires spécialisés et généraux: constats et analyses. Les Sciences de l'éducation-Pour l'Ére nouvelle, 3(38), 33-51. doi: 10.3917/ lsdle.383.0033.

Boilevin, J. (2005). Enseigner les physiques par situation problème ou par problème ouvert. Aster, (40), 13-38. Recuperado de http://ife.enslyon.fr/publications/edition-electronique/ aster/RA040.pdf

Bozu, Z., \& Canto, P. (2009). El profesorado universitario en la sociedad del conocimiento: competencias profesionales docentes. Revista de Formación e Innovación Educativa Universitaria, 2(2), 87-97. Recuperado de http://webs.uvigo.es/refiedu/Refiedu/ Vol2_2/arti_2_2_4.pdf

Chadwick, C. (2001). La psicología de aprendizaje del enfoque constructivista. Revista Latinoamericana de Estudios Educativos, 31(4). 111-126.

Dilworth, R. L. (1998). Action learning in a nutshell. Performance Improvement Quarterly, 11(1), 28-43.

Dotlich, D., \& Noel, J. (1998). Action learning: How the world's top companies are re-creating heir leaders and themselves. San Francisco: Jossey-Bass.
Fabre, M. (2005). Deux sources de l'épistémologie des problèmes: Dewey et Bachelard. Les Sciences de l'éducation-Pour l'Ére nouvelle, 3(38), 5367. doi: 10.3917/1sdle.383.0053.

. (2006). Chapitre1. Qu'est-ce que problématiser? L'apport de John Dewey. En M. Fabre \& E. Vellas (Ed.), Situations de formation et problématisation (pp. 15-30). De Boeck Supérieur. Recuperado de http://www.cairn. info/situations-de-formation-et-problematisation---page-15.htm

Freire, P. (1983). Extensão ou comunicação? (7ª ed.). São Paulo: Paz e Terra.

\section{(2006). Pedagogia da autonomia:} saberes necessários (33a. ed.). São Paulo: Paz e

Huber, G. (2008). Aprendizaje activo y metodologías educativas - Active learning and Methods of teaching. Revista de Educación, numero extraordinario, 59-81. Recuperado de http://www.revistaeducacion.mec.es/re2008/ re2008_04.pdf

Kristensen, E., Sander-Regier, R., Merhy, B. \& McColl, T. (2007). Enseigner à l'Université d'Ottawa: Guide pour les professeurs et les assistants à l'enseignement (50a. ed.). Ottawa: UOTTAWA. Recuperado de http://www. sante.uottawa.ca/pdf/g_profl.pdf

Marquardt, M. (1999). Action learning in action: Transforming problems and people for world-class organizational learning. Palo Alto: Davies-Black Publishing. 
Marquardt, M. (2004). Optimizing the power of action learning. Palo Alto: Davies-Black Publishing.

Marquardt, M., \& Waddill, D. (2004). The Power of Learning in Action Learning: A Conceptual Analysis of How the Five Schools of Adult Learning Theories Are Incorporated within the Practice of Action Learning. Action Learning Research and Practice, 1(2), 185202. Recuperado de: file:///media/Datos/ Downloads/1476733042000264146.pdf

Minardi, S., Siqueira, R., Girardi, J., Morais, N., Almeida, C., Pinto, C., Moreira, T. \& Amaral, L. (2008). Metodologias de ensino-aprendizagem na formação profissional em saúde: debates atuais. Ciência e Saúde Coletiva,13 (Supl. 2), 2133-2144. Recuperado de http://www.scielo.br/pdf/csc/v13s2/v13s2a18.pdf

Mumford, A. (1991).Learning in action. Personnel Management, 23(7): 34-40.

Orange, C. (2005). Problème et problematisation dans l'enseignement scientifique. Aster, (40), 3-10. Recuperado de http://ife.ens-lyon.fr/ publications/edition-electronique/aster/ RA040.pdf

Ormorond, J. (2008). Aprendizaje Humano (4a ed.). Madrid: Pearson Educación.

Pérez, A. (1988). El pensamiento práctico del profesor/a. Implicaciones en la formación del profesorado. En A. Villa (Ed.), Perspectivas y problemas de la función docente (pp. 128-149). Madrid: Narcea.

Pedler, M., (Ed.). (1997). Action learning in practice. Aldershot: Gower.

Rumelhard, G. (2005). Problématisation et concept de paradigme. Aproche épistémologique, psychologique, sociologique. Aster, (40), 205224. Recuperado de http://ife.ens-lyon.fr/ publications/edition-electronique/aster/ RA040.pdf

Saviani, D. (1996). Educação do senso comum à consciência filosófica (11a. ed.). Campinas: Autores Associados.

Schawartz, S., \& Pollishuke, M. (1998). Aprendizaje activo: Una organización de la clase centrada en el alumnado. Madrid: Narcea.

Shuell, T. (1986). Cognitive conceptions of learning. Review of Educational Research, (56). 411-436.

St-Jean, M. (2001). L'apprentissage actif. Comment des proffesseursengagentleursétudiantsdan sunapprentissageactif. Bulletin CÉFES, 4,1. Recuperado de http://www.cefes.umontreal. $\mathrm{ca} / \mathrm{ressources/bulletins/BulletinCEFES}$ no4.pdf

Uniminuto. (2004). Modelo Educativo Uniminuto. Bogotá: Uniminuto.

Vygotski, L. (1989). El desarrollo de los procesos psicológicos superiores. Barcelona: Crítica.

Weinstein, K. (1995). Action learning: A journey in discovery and development. Londres: Harper Collins.

Westbrook, R. (1993). John Dewey. Perspectivas: revista trimestral de educación comparada, 23(1-2). 289-305.

Zanotto, M., \& Rose, T. (2003). Problematizar a própria realidade: análise de uma experiência de formação. Revista Educação e Pesquisa, 29 (1), 45-54. Recuperado de http://www.scielo. br/pdf/ep/v29n1/a04v29n1.pdf 